\title{
Radiology double reads
}

\section{Anik Sahni, Ramin Khorasani}

Department of Radiology, Center for Evidence-Based Imaging, Brigham and Women's Hospital, Harvard Medical School, Boston, Massachusetts, USA

\section{Correspondence to}

Dr V Anik Sahni, Department of Radiology, Center for EvidenceBased Imaging, Brigham and Women's Hospital, 20 Kent Street, 2nd Floor, Boston, MA 02445, USA vsahni@partners.org

Accepted 2 May 2016 Published Online First 26 May 2016

\section{SLinked}

- http://dx.doi.org/10.1136/ bmjqs-2015-004536

\section{CrossMark}

To cite: Sahni VA,

Khorasani R. BMJ Qual Saf 2016:25:569-571.
Diagnostic errors in radiology reports can result in patient harm, lead to unnecessary additional testing or interventions, or otherwise diminish the quality of care delivery. ${ }^{1}{ }^{2}$ In this issue, Lauritzen et al retrospectively evaluated the impact of a quality assurance process using double reads on changes made to abdominal CT reports requested by referring surgeons. ${ }^{3}$ The authors address an important topic in this insightful study highlighting the challenges and opportunities related to improving radiology report quality. The authors analysed 1071 consecutive abdominal CT reports on patients referred by surgeons from five public Norwegian hospitals double read by two consultant radiologists over a 17 -month period. The request for the second read was initiated at the judgement of the primary radiologist reader who could have been an inexperienced radiologist, an experienced general radiologist, an abdominal radiology subspecialist or a subspecialist in another organ system. Preliminary and final reports were compared for changes and the clinical importance of these changes were rated, on a five-point scale, by at least two gastrointestinal surgeons each with at least 10 years of experience. In addition, the severity of any changes was classified as increased, decreased or unchanged. Of these reports, 146 (14\%) were identified as containing clinically important changes; 3 critical, 35 major and 108 intermediate. The severity of the changes was increased in 118 (81\%) of reports with clinically important changes. Multivariate analysis demonstrated that important changes were more frequent when the examination was urgent or when the second reader was an abdominal radiologist and less frequent when the first reader was an abdominal radiologist.

The primary goal of the radiologist is to provide the referring provider with an actionable imaging report ${ }^{4}$ that conveys the pertinent findings identified in an appropriately and correctly performed imaging examination and communicate these findings unambiguously and in a timely manner. Any deviation from this primary objective results in a disservice to our patients and undermines our value as clinicians. The identification of abnormal findings in an imaging examination and their accurate interpretation is the key component of the integrity of an imaging report.

Despite our best efforts, diagnostic errors occur at regular frequency within radiology reports. ${ }^{5}$ The contributing causes of radiology errors are myriad, but their presence behoves the radiological community to implement quality improvement and quality assurance processes to minimise the presence and impact of errors within imaging reports.

A double reading process as described by the authors of this article is one model to diminish diagnostic errors. Such an approach is not conceptually limited to radiology. Presented with the same patient, two surgeons may have differing opinions on treatment options. There are examples of multidisciplinary clinical decision-making processes in cancer care (eg, care for patients with pancreatic cancer typically involves the collaboration of surgeons, medical and radiation oncologists, gastroenterologists, radiologists and pathologists). A major challenge in implementation of such approaches is coordinating and accounting for the needed resources. Lauritzen et al describe an ad hoc prospective double read process initiated by the primary reader presumably due to his/her uncertainty about the presence or clinical relevance of specific imaging findings. By the authors' own admission, the double read process as described consumes a substantial amount (20\%-25\%) of their professional resources. It is also interesting that it is the second reader who finalises the report, with a mean delay of approximately $20 \mathrm{~h}$ from the preliminary interpretation. In large academic radiology practices in the USA and Canada seeking a consultation from a second reader is 
quite common (although much less common than the $39 \%$ frequency of double reads in the Lauritzen et al study). However, finalising the report is almost always the primary reader's responsibility. Such an informal approach to double reads most likely diminishes the delay to finalise the report compared with Lauritzen et al, though data about its frequency, impact on turnaround times or the quality of the report are lacking and difficult to assess.

Another model to improve the quality of radiology reports is increasing subspecialisation. For example, abdominal radiology subspecialists are more likely to adhere to evidence-based guidelines for management of renal masses than general radiologists or radiologists in other subspecialties. ${ }^{6}$ Conceptually, subspecialisation in radiology follows similar approaches to medical and surgical subspecialties. Luaritzen et al's findings support such an approach for quality improvement. Clinically important changes in the final reports were less common when the primary reader was an abdominal radiologist. The major drawback to such as approach is the timely availability of subspecialty resources. However, given the relative maturity of health information technology (IT) solutions for digital imaging, such as Picture Archiving and Communication Systems (PACS), it may be practical to create workflows whereby the majority or even all abdominal CTs referred by surgeons as outpatients or inpatients are interpreted by abdominal radiology subspecialists. That is indeed the standard of care at our institution. Alternatively, such referrals may be limited to urgent requests to lessen the burden on subspecialty resources. It would indeed be an interesting study to compare the value of the double read process of Lauritzen et al's study (eg, quality and timeliness of final radiology reports and the radiologist resources needed) to a model where abdominal radiologists are the primary readers, supplemented by a $2 \%-3 \%$ random peer review quality assurance process (a typical subspecialty model of care in the USA).

Other factors that may be seen as obstacles to the institution of a double read workflow include delays in issuing the final report, biased opinions on the second read based on interpersonal relationships and communication and the potential medicolegal implications of the second reader changing the primary readers report.

Many departments, especially in the USA, have instituted a peer review process as a quality improvement initiative. ${ }^{7}$ The main driving force initially behind these programmes was to fulfil accreditation requirements for certain regulatory bodies such as the American College of Radiology. Typically, such review is performed on finalised reports and occurs on a smaller proportion of reports than described by Lauritzan et al (approximately $2 \%-5 \%$ vs $39 \%$ of CT reports in Norway). In our experience the retrieval of major clinically relevant discrepancies during random peer review has been very limited. However, the radiologists in our practice are encouraged to peer review reports finalised the same day so as to have the ability to potentially change contemporary management if a diagnostic error is uncovered.

No one solution exists to the reduction in diagnostic errors in radiology reports. Where radiologist time and resources are limited and double reading may not be possible or limited, information technology tools can be used to improve accuracy and consistency. The burgeoning field of decision-support tools available to the radiologist at the workstation at the time of reporting is one such example. These tools provide the radiologist with applications to help accurately identify abnormalities, correctly interpret findings and provide consistent follow-up recommendations. ${ }^{8}{ }^{9}$ The success of these tools is dependent on embedding them into the radiologists' workflow so they are seamlessly and easily accessed. This can be done by adding the software as 'plug-ins' to PACS workstations, embedding hyperlinks into reports templates to effortlessly access information resources and integrating follow-up recommendations into speech recognition software. ${ }^{4}$

In conclusion the concept of imaging double reads as shown by Lauritzen et al has the potential to reduce diagnostic errors but requires sizeable resources to institute. Future studies would be needed to assess the role of subspecialisation, workflow optimisation and use of health IT solutions such as computer-aided detection/diagnosis and decision support tools to improve the performance of radiologists. Although we applaud the authors for meticulous collection and analysis of data to support the notion of double reads as a primary means of improving the quality of radiology reports, further studies are needed to assess and compare the impact of other interventions on report quality. Although limited double reads, in particular situations, such as urgent scans, may be one solution, as suggested by the authors, subspecialisation and judicious use of information technology tools may provide radiologists with new and developing resources to reduce diagnostic errors and improve patient care.

Competing interests None declared.

Provenance and peer review Commissioned; internally peer reviewed.

\section{REFERENCES}

1 Donald JJ, Barnard SA. Common patterns in 558 diagnostic radiology errors. J Med Imaging Radiat Oncol 2012;56:173-8.

2 Pinto A, Reginelli A, Pinto F, et al. Errors in imaging patients in the emergency setting. Br J Radiol 2016;89:20150914.

3 Lauritzen PM, Andersen JG, Stokke MV, et al. Radiologistinitiated double reading of abdominal computed tomography: retrospective analysis of the clinical importance of changes to radiology reports. BMJ Qual Saf 2016;25:595-603. 
4 Sahni VA, Khorasani R. The actionable imaging report. Abdom Radiol 2016;41:429-43.

5 Kim YW, Mansfield LT. Fool me twice: delayed diagnosis in radiology with emphasis on perpetuated errors. AJR Am J Roentgenol 2014;202:465-70.

6 Maehara CK, Silverman SG, Lacson R, et al. Journal club: renal masses detected at abdominal CT: radiologists' adherence to guidelines regarding management recommendations and communication of critical results. AJR Am J Roentgenol 2014;203:828-34.
7 Brook OR, Romero J, Brook A, et al. The complementary nature of peer review and quality assurance data collection. Radiology 2015;274:221-9.

8 Firmino M, Morais AH, Mendoça RM, et al. Computer-aided detection system for lung cancer in computed tomography scans: review and future prospects. Biomed Eng Online 2014;13:41.

9 Wang S, Burtt K, Turkbey B, et al. Computer aided-diagnosis of prostate cancer on multiparametric MRI: a technical review of current research. Biomed Res Int 2014;2014:789561. 